Revue internationale P.M.E.

Économie et gestion de la petite et moyenne entreprise

\title{
De la veille technologique au pilotage stratégique
}

\section{Michel Marchesnay}

Volume 1, numéro 3-4, 1988

URI : https://id.erudit.org/iderudit/1007889ar

DOI : https://doi.org/10.7202/1007889ar

Aller au sommaire du numéro

Éditeur(s)

Presses de l’Université du Québec

ISSN

0776-5436 (imprimé)

1918-9699 (numérique)

Découvrir la revue

Citer cette note

Marchesnay, M. (1988). De la veille technologique au pilotage stratégique.

Revue internationale P.M.E., 1(3-4), 335-340. https://doi.org/10.7202/1007889ar d'utilisation que vous pouvez consulter en ligne.

https://apropos.erudit.org/fr/usagers/politique-dutilisation/ 


\section{De la veille technologique au pilotage stratégique}

Michel Marchesnay

ERFI

Université de Montpellier

Une note précédente était consacrée à l'importance de la technologie pour la P.M.E. Il s'agit ici, en quelques pages, de montrer comment est abordé le problème de la surveillance des modifications technologiques dans l'environnement, et, plus généralement, du pilotage stratégique, c'est-à-dire de la régulation des manoeuvres stratégiques, grâce à un système d'informations sur l'environnement adéquat. Nous nous appuierons sur la lecture d'ouvrages récents en français qui traitent de ce problème, quitte à inclure, in fine, quelques observations sur des articles récents en langue anglaise qui nous semblent exprimer un point de vue intéressant, tout en ouvrant des perspectives de recherche fécondes. Toutefois, reconnaissons d'emblée que notre sentiment dominant vis-à-vis de la plupart des travaux évoqués ci-dessous est celui d'une forte insatisfaction, au regard de la façon dont est traité le problème spécifique de laP.M.E. Par contre, il faut convenir que ces travaux ouvrent de la sorte des voies et avenues de recherche sur le domaine de la veille technologique et le pilotage stratégique de la P.M.E.

L'ouvrage incontestablement le plus intéressant pour le gestionnaire, y compris le propriétaire-dirigeant de petite unité, est celui de Martinet et Ribault (1989). Ces deux auteurs sont consultants et leur méthodologie respire ce pragmatisme inhérentà l'implantation d'un système d'information. De ce point de vue, la première partie semble plus appropriée à la résolution des besoins des P.M.E. Les auteurs distinguent quatre grands types de veille: technologique, concurrentielle, commerciale et environnementale. Chacune de ces veilles nécessite un système d'information approprié, impliquant l'accès à des sources dont le crible sera fonction de la détection des besoins réels en information, au travers d'indicateurs. La cueillette d'information est donc conditionnée par son usage futur - notamment la recherche d'innovation de produits. Au total, l'entreprise doit se constituer un observatoire de l'environnement. L'intérêt majeur du livre réside dans les nombreux tableaux et schémas (même si certains sont fort contestables) qui servent à guider le montage d'un tel observatoire. 
Les ouvrages suivants n'obéissent pas à une telle ambition pragmatique. Il s'agit plutôt d'oeuvres académique, dont l'objet est de sensibiliser enseignants et chercheurs à la stratégie technologique. L'ouvrage de Dussauge et Ramanantsoa (1987), professeurs à HEC-Paris, est révélateur d'une tendance que l'on retrouve dans l'ouvrage collectif de la même école «Strategor» : «Stratégie, structure, décision, identité» (1988). La technologie est définie (p. 19) sur les critères suivants : une «entreprise» (de recherche), une production matérielle d'objets, une démarche, une «combinaison des technologies», soit un "procédé» - un ensemble de procédés -permettant, après une démarche explicite ou implicite de recherche ou d'amélioration des techniques de base ou d'applications de connaissances scientifiques, d'envisager une production industrielle. La stratégie, elle, est définie de la façon suivante (p.43): «Élaborer la stratégie de l'entreprise, c'est choisir les domaines d'activité dans lesquels elle entend être présente et allouer des ressources de façon à ce qu'elle s'y maintienne et s'y développe». L'apport essentiel des auteurs est alors double. Premièrement, ce que nous appellerons, personnellement, «le panier des technologies maitrisées par l'entreprise» va constituer son métier. Or, beaucoup trop d'entreprises ont eu tendance à négliger le métier de base pour s'aventurer dans des «terrae incognitae», dans des activités dont on maîtrisait mal le savoir-faire, même si l'on avait le savoir-vendre. On constate donc un recentrage sur le métier : appréciation qui mérite d'être nuancée, à la lumière des opérations actuelles de croissance externe, mûes par la logique plus financière qu'industrielle... et par le fait que les auteurs éprouvent eux-mêmes quelques difficultés à cerner la notion de métier! Il n'en reste pas moins vrai que, pour les P.M.E., leur atout réside, pour l'essentiel, dans la maîtrise de leur métier. Et c'est là qu'arrive le second apport des auteurs : le métier est partie intégrante de l'identité de l'entreprise (p.207). Les individuss'identifient àl'entreprise de par leur métier propre, le métier de l'entreprise, et, enfin, les éléments de l'«imaginaire organisationnel» (ce que d'autres appellent la culture de l'entreprise). L'ouvrage séduit et irrite. Il est une belle construction intellectuelle où les concepts se révèlent parfois insaisissables. Surtout, laP.M.E. est bien absente despréoccupations des auteurs, alors que ces problèmes de veille technologique y sont préoccupants. Au demeurant, les auteurs ajoutent une annexe consacrée à l'étude de P.M.I. nouvelles, innovatrices et de haute technologie (p. 217 s.). Les auteurs soulignent alors l'inadéquation des modèles stratégiques courants pour de telles entreprises : il est en effet bien difficile de positionner de telles activités en devenir sur des marchés à constituer. Les auteurs se contentent alors de quelques mises en garde pour les dirigeants, avant d'ajouter (p. 223) : «La réussite d'une jeune P.M.I. passe, plus que toute autre entreprise (sic), par un jeu relationnel important auquel doit être rompu ou se plier le chef d'entreprise... Il conviendrait de ne pas oublier les échanges technologiques qui peuvent être réalisés avec certains groupes importants et qui souvent se couplent avec des répartitions de marché hautement profitables au plus petit». Vision irénique des relations entre «gros» et «petits» dont nous laissons l'entière responsabilité aux auteurs...

L'ouvrage collectif du G.E.S.T. (1986), plus ancien, a contribué à vulgariser la notion de "grappes technologiques». L'intérêt est de montrer que «la technologie 
(est) au centre de la stratégie» (p. 20), et que bien des «technologies» ne sont que le fruit de bricolages dans des ateliers de petite entreprise (p. 21). Comme l'indiquent les auteurs (p. 27), «le terme de grappe technologique désigne une collection d'activités liées entre elles par une essence technologique commune. La grappe est formée d'un ensemble d'axes de valorisation, partant de la technologie pour aboutir à des produits sur des marchés. On pourra ironiser sur cette «essence technologique» qui a un parfum théologique; il n'en reste pas moins que cette notion de grappe s'avère pertinente pour aborder les problèmes suivants :

- Quelle est la technologie de base dans l'environnement technologique de l'entreprise ? Comment s'articule-t-elle et se diffuse-t-elle jusqu'au produit final ?

- Comment se combinent les technologies? Les auteurs parlent de combinaison par «agglomération», par «percolation», par «théorisation» (p. 55 et s.).

- Comment se fait le changement de technologie et la diffusion au sein d'une grappe ? Les auteurs parlent de stratégies génériques, comme axe de développement de la diversification. On en trouve un excellent exemple dans le cas Arc-en-Ciel (ERFI, 1989), appliqué à une P.M.E.

- Comment les entreprises collaborent-elles entre elles au sein d'une grappe technologique ? On débouche ici sur la notion de réseaux, tant à la mode.

Enfin, pour ceux qui lisent l'espagnol, on ne saurait trop conseiller la lecture de l'ouvrage de Fernandez Sanchez et Fernandez Casariego (1989) qui traite du management stratégique de la technologie. Cet ouvrage constitue une remarquable synthèse de la littérature sur le sujet, et mériterait une traduction en français.

Les ouvrages que nous voudrions présenter maintenant abordent le problème plus général de la veille et du pilotage stratégiques. Ce problème se résout, en définitive, par la constitution d'un système d'information approprié à la surveillance systématique de l'environnement, quelle que soit la taille de l'entreprise.

Marie-José Avenier (1985, nouvelle édition 1988) définit d'emblée le pilotage à la fois par le fait que dans l'entreprise on se fixe des objectifs généraux à atteindre, et par la conduite de l'entreprise vers la réalisation de ces objectifs». Par modèle de pilotage, l'auteur entend «un concept et un schéma de mise en oeuvre de ce concept» (p. 11). Defait, il s'agit de concilier une démarche stratégique «rationaliste» de planification et une approche «incrémentaliste» (d' «heuristique», selon l'auteur), au travers d'une planification à la fois «intégrative» (qui «fonde le développement de l'entreprise sur l'exploitation de ses forces actuelles en recherchant une meilleure adaptation de ses diverses activités») et une planification adaptative (qui «vise à assurer une meilleure adaptation des activités de l'entreprise à son environnement»). La conciliation semble particulièrement indispensable à l'auteur pour les P.M.E. françaises, dans lesquelles (p. 20) «on ignore tout de l'approche rationaliste (son objet, sa philosophie, ses méthodes, ses outils) et où la diffusion de l'approche heuristique risque d'être rapide et extrêmement néfaste... Elle risque de les conforter dans leur attitude de pilotage à vue». Mais, par la suite, l'auteur va s'appuyer sur 
l'expérience de cinq grandes entreprises ou groupes industriels pour conforter sa conviction de la nécessité d'une approche intégrative. Celle-ci se concrétise dans le système de pilotage stratégique (SPS), défini (p. 138) comme «un ensemble cohérent et évolutif de procédures destinés à la mise en oeuvre du pilotage stratégique de l'entreprise. «Il y a cinq types de processus (d'information, de réflexion stratégique, de programmation pluriannuelle, de budgétisation et de contrôle) dans le management stratégique, auxquels on ajoute, dans le pilotage stratégique, des processus «de formation, de motivation et d'urgence». La conception d'un SPS comporte alors trois phases (p. 152) : définition d'une structure stratégique des activités (segmentation stratégique), détermination d'un cadre stratégique global, spécification des sousprocessus relatifs à chaque unité».

Le mérite de cette approche réside dans le formalisme auquel on s'astreint; c'est aussi un risque. Le refus de toute contingence conduit à poser le problème du pilotage stratégique de façon identique pour une petite ou pour une grande entreprise. Une telle attitude méconnaît l'extrême variété de situations en P.M.E. autant que la spécificité du processus de prise de décision stratégique (pour le considérer comme «irrationnel»). Par exemple, l'auteur affirme péremptoirement (p. 199) : «Comme l'atout majeur d'une entreprise de petite taille est sa mobilité, les caractéristiques variables de son système de pilotage seront fixées de façon à renforcer cette propriété : un degré de décentralisation élevé, un degré de formalisme faible, des calendriers, une attitude plutôt (sic) téméraire vis-à-vis du risque». Cette proposition révèle un degré élevé de normativité, fondée sur des arguments infalsifiables : il serait aisé de reprendre la phrase en émettant des propositions contraires, tout aussi faiblement étayées par des faits justificatifs. De même, page 212, une figure 23 met en relation les «attributs d'une entreprise» et les «caractéristiques d'un système de pilotage» : on constate que la petite taille ne joue aucun rôle, sauf sur le degré de formalisme! Fort heureusement, comme le note avec bon sens l'un des auteurs de Strategor à propos des modèles de portefeuille d'activités : «Peu concernées par ces modèles les [P.M.E.] ont concentré leurs efforts sur la gestion quotidienne et opérationnelle de leur activité, acquérant ainsi une compétence distinctive qui est devenue un facteurclé de succès dans la situation économique présente» (p. 138). De quoi remettre à l'honneur le pragmatisme du dirigeant, même affublé du vocable d' «heuristique»...

L'ouvrage de Lesca (1986) aborde le problème du système d'information nécessaire à la conduite du management stratégique. La qualité du management stratégique est censée être fonction de la qualité de l'information récoltée et exploitée. L'entreprise doit s'appuyer sur une «formule stratégique» (comprenant le marché, la technologie et l'organisation) et sur les facteurs de «force motrice» (p. 51 et s.), soit : les produits, les besoins, la technologie, le savoir-faire, les méthodes de vente et de distribution, les ressources naturelles, la taille, la rentabilité. Dans cette tâche de veille de l'environnement, le dirigeant, et son style de direction, joue un rôle déterminant (p. 62 et s.); le problème, sorte de "pont-aux-ânes» du management des systèmes d'information, est alors de savoir si cette activité de veille doit être assurée par un service spécialisé ou diluée dans l'ensemble de l'organisation. Reconnaissons 
que la réponse n'est pas claire et qu'elle pose problème dans une P.M.E. : en clair, est-ce au dirigeant d'assumer cette activité de surveillance ? Sinon, comment contrôler que celle-ci soit bien faite par les collaborateurs ? Lesca nous donne l'exemple d'une P.M.I. (p. 94 et s.), appelée Transflux pour les besoins de la cause, engluée dans un service d'information stratégique. De même, la P.M.E. a-t-elle intérêt à «faire sa veille», ou à la «faire faire», par exemple par des consultants extérieurs ? La réponse de l'auteur est très prudente. Au demeurant, l'un des mérites de cet ouvrage est de faire constamment référence à des P.M.E. grenobloises, dans lesquelles l'auteur semble avoir acquis une expérience de terrain : cela n'est pas si fréquent dans les ouvrages touchant au management stratégique!

Au total, une brève recension d'ouvrages français sur ce problème de la veille et du pilotage laisse apparaître une forte normativité : un champ de recherche empirique semble bien ouvert aux chercheurs en P.M.E. Quelques articles récents, notamment américains, confirment ce sentiment. Ainsi, dans l'un des derniers numéros de JSBM (juillet 88), Smeltzer, Fann et Nikolaisen ont étudié les pratiques de «scrutation» de l'environnement des petites entreprises. En distinguant trois types de «scrutation» (irrégulier, périodique et continu) et en interrogeant 88 propriétairesdirigeants d'entreprises de moins de 30 salariés, on confirme que la fonction est concentrée dans les mains du propriétaire-dirigeant et qu'elle est d'autant moins formalisée que l'entreprise est plus petite. L'environnement est le plus souvent ramené au seul marché, les sources extérieures d'information sont peu utilisées, et, en fait, on s'appuie sur les personnes avec lesquelles on est en interaction (ce que nous avons appelé, dans le système de gestion de la P.E., le système d'interaction). Il va de soi que moins l'environnement apparaît stable, plus cette fonction de «scrutation» apparaît importante.

Cette dernière impression est confirmée dans une livraison récente de Strategic Management Journal (janvier 1989). Covin et Slevinétudient le management stratégique dans les environnements «hostiles» et «bénins». A partir d'un échantillon définitif de 133 entreprises, de 5 à 500 salariés, appartenant à 25 secteurs d'activité industrielle, les auteurs montrent que les firmes ont des réponses stratégiques différenciées selon la nature de l'environnement. Les entreprises à performance élevée en environnement hostile disposent d'une structure organique, une «posture stratégique compétitive» (définie comme «une orientation sur la compétitivité globale»), un profil concurrentiel axé sur le long terme, une approche d'administration centrée sur les buts, des prix produits-services élevés et un souci de maintenir une conscience des grandes tendances de l'industrie. Dans les environnements «bénins», on adoptera une structure mécaniste, une posture stratégique plus conservatrice, une démarche à plus court terme, on améliorera les produits existants et on recherchera la dépendance à l'égard d'un gros client. Ces résultats pourront apparaître sans surprise et entachés de l'inévitable «péché originel» de la recherche empirique, surtout par questionnaire postal, à savoir la subjectivité des réponses (qu'est-ce au juste qu'un «environnement hostile» ?), mais ils confirment le rôle de la veille stratégique dans les P.M.E. 
Dans un article récent de la revue Harvard-1'Expansion (hiver 88-89), repris d'un article paru dans la HBR (mai 88) qui précise le rôle des conseillers et de l'entourage de l'entrepreneur, Stevenson et Sahlman notent avec pertinence : «Il va sans dire qu'il faut lire avec avidité et posséder à l'intérieur et à l'extérieur de la profession, un vaste réseau d'amis à qui demander comment ils s'y sont pris pour résoudre tel ou tel problème, quels experts ils ont sollicités et quels ont été leurs succès et leurs échecs. En procédant ainsi, vous acquerrez de l'expérience avant d'en avoir besoin et pourrez écarter plus d'un mauvais conseiller» (p. 21).

Au total, la petite entreprise se trouve, plus que jamais elle ne l'a été sans doute, ouverte sur son environnement. S'il appartient au propriétaire-dirigeant de décider, et bien souvent de décider seul, il ne peut, pour le faire, s'enfermer dans sa tour d'ivoire. A un degré croissant, la P.M.E. tisse un tissu de relations externes qui viennent orienter et conforter le sens des investigations et la nature des informations liées à la veille stratégique. En ce sens, la conception d'un système de pilotage stratégique adapté aux besoins de la petite organisation reste encore largement à faire.

\section{BIBLIOGRAPHIE}

Avenier, M.J., (1988), Le pilotage stratégique de l'entreprise, Éditions du CNRS, Paris, $2^{\circ}$ édition.

Covin, J.G. et Slevin, D.P., (1989), «Strategic management of small firms in hostile and benign environments», Strategic Management Journal, vol. 10, $\mathrm{n}^{\circ}$ 1, janvier-février, pp. 75-87.

Dussauge, P. et Ramanantsoa, B., (1987), Technologie et stratégie d'entreprise, Paris, McGraw-Hill.

Fernandez Sanchez, E. et Fernandez Casariego, Z., (1988), Manual de direccion estraegica de la tecnologia, Ariel Economia, Barcelona.

G.E.S.T., (1986), Grappes technologiques. Les nouvelles stratégies d'entreprise, Paris, McGraw-Hill.

ERFI, (1989), Cas et solutions de stragégie en PME, Paris, Chotard.

Lesca, H., (1986), Système d'information pour le management stratégique de l'entreprise, Paris, McGraw-Hill.

Martinet, B. et Ribault, J.-M., (1988), La veille technologique, concurrentielle et commerciale, Paris, Les Éditions d'Organisation 1988.

Smeltzer, L.R., Fann, G.L. et Nikolaisen, V.N., (1988), «Environmental scanning practices in small business», Journal of Small Business Management, vol. 26, $\mathrm{n}^{\circ} 3$, juillet.

Stevenson, H.H. et Sahlman, W.A., (1988), «Les petites entreprises face à leurs conseillers», Harvard-l' Expansion, $n^{\circ}$ 51, hiver 89. 